\title{
A posteriori error bounds for fully-discrete $h p$-discontinuous Galerkin timestepping methods for parabolic problems
}

\author{
Emmanuil H. Georgoulis ${ }^{1,2}$. Omar Lakkis ${ }^{3}$. Thomas P. Wihler ${ }^{4}$
}

Received: 22 August 2017 / Revised: 9 August 2020 / Accepted: 30 January 2021 /

Published online: 14 April 2021

(C) The Author(s) 2021

\begin{abstract}
We consider fully discrete time-space approximations of abstract linear parabolic partial differential equations (PDEs) consisting of an $h p$-version discontinuous Galerkin (DG) time stepping scheme in conjunction with standard (conforming) Galerkin discretizations in space. We derive abstract computable a posteriori error bounds resulting, for instance, in concrete bounds in $\mathrm{L}_{\infty}\left(I ; \mathrm{L}_{2}(\Omega)\right)$ - and $\mathrm{L}_{2}\left(I ; \mathrm{H}^{1}(\Omega)\right)$-type norms when $I$ is the temporal and $\Omega$ the spatial domain for the PDE. We base our methodology for the analysis on a novel space-time reconstruction approach. Our approach is flexible as it works for any type of elliptic error estimator and leaves their choice to the user. It also exhibits mesh-change estimators in a clear and concise way. We also show how our approach allows the derivation of such bounds in the $\mathrm{H}^{1}\left(I ; \mathrm{H}^{-1}(\Omega)\right)$ norm.
\end{abstract}

Mathematics Subject Classification 65M60 $\cdot 65 \mathrm{M} 15 \cdot 65 \mathrm{M} 50$

Emmanuil H. Georgoulis

Emmanuil.Georgoulis@le.ac.uk

Omar Lakkis

lakkis.o.maths@gmail.com

Thomas P. Wihler

wihler@math.unibe.ch

1 Department of Mathematics, University of Leicester, Leicester LE1 7RH, UK

2 Department of Mathematics, School of Applied Mathematical and Physical Sciences, National Technical University of Athens, Zografou 15780, Greece

3 Department of Mathematics, University of Sussex, Falmer, Brighton GB-BN1 9QH, England, UK

4 Mathematisches Institut, Universität Bern, Sidlerstrasse 5, 3012 Bern, Switzerland 


\section{Introduction}

Adaptive numerical methods have been shown to provide accurate and efficient numerical treatment of evolution PDEs thanks to their properties for localized mesh resolution especially in the context of moving fronts, interfaces, singularities, or layers (both boundary and interior). Such numerical methods predominantly admit spatial discretizations of variational type, e.g., finite element methods (FEMs), which allow for general, possibly unstructured, dynamic mesh modification. FEMs are also ideally suited for deriving mathematically rigorous a posteriori bounds, owing to their variational nature.

Some of the classical works on adaptive finite element methods for parabolic problems [10-14] are based on discontinuous Galerkin (DG) time stepping combined with FEM in space, and proving a posteriori bounds in various norms using duality techniques. The key motivation in using DG in time, which is also of variational type, is that it naturally allows for spatially-local-time stepping, i.e. different time step sizes in different parts of the spatial domain $[10,15,21,25]$. This classical, but as of yet undeveloped in full, concept of local adaptivity in both space and time has the potential of delivering substantial computational savings and even complexity reduction.

In addition to the ability of Galerkin time marching schemes to employ locally different time step sizes, their variational character also allows for arbitrary variations in the local approximation orders. They can therefore be cast naturally into the framework of $h p$-approximation schemes. In the context of parabolic PDEs, $h p$-version time marching methods can be used, for instance, to resolve an initial layer in the (otherwise smooth) solution at high algebraic or even exponential rates of convergence, see, e.g., the works $[33,34,39]$ on linear parabolic PDEs, and also [29,30] which employ a combination of $h p$-version time stepping with suitable wavelet spatial discretizations to yield a log-linear complexity algorithm for nonlocal evolution processes involving pseudo-differential operators. Additionally, we note the numerical analysis of highdimensional parabolic problems using sparse grids in space; see [38].

More recent results on rigorous a posteriori bounds for parabolic problems have focused on extending the paradigm of the reliable and efficient a posteriori error analysis of elliptic problems to the parabolic case [31,36,37]. Such works typically involve basic low-order time stepping schemes combined with various types of FEM in space. A posteriori error bounds for DG time-stepping methods have also appeared in the last few years; we point to $[22,27,35]$ which are based on the reconstruction technique, to $[16,17]$ which employ an equilibrated flux approach, or to [19] which presents a provably convergent adaptive algorithm for a residual-type a posteriori estimator.

In this paper, we present a posteriori error bounds for an $h p$-version DG-in-time and conforming Galerkin discretization in space method for both $\mathrm{L}_{\infty}\left(I ; \mathrm{L}_{2}\right)$ - and $\mathrm{L}_{2}\left(I ; \mathrm{H}^{1}\right)$-norm errors separately, allowing for what appears to be optimal order in each case. The key idea is the use of suitable reconstruction frameworks to derive a perturbed PDE for the reconstructed error of the numerical method; a posteriori error bounds are then deduced using PDE stability properties, cf. [1,23,26,27]. Our approach is based on new space and space-time reconstructions which are built on the combination of respective ideas for DG-time stepping methods [27,35] and elliptic 
reconstruction $[23,26]$ to the fully-discrete setting. To that end, the key challenge of constructing a globally time-continuous reconstruction in the presence of mesh modification between time-steps is addressed by first reconstructing onto the solution space with respect to the spatial variables via a novel elliptic reconstruction definition, given in (3.14), which is a modification of the one in [23]. In particular, the new proposed elliptic reconstruction takes into account the effect of mesh-change.

Our results are closely related, however, with important departures, to those of $[16,17,19]$. In particular, the new reconstructions defined below allow for the derivation of a posteriori upper error bounds for each of the following norms $\mathrm{L}_{2}(I ; \mathscr{X})$ (Theorem 4.4) and $\mathrm{H}^{1}\left(I ; \mathscr{X}^{\prime}\right)$ (Sect. 6) separately; the Hilbert space $\mathscr{X}$ is the domain of a self-adjoint uniformly elliptic operator $\mathscr{A}$ (see Sect. 2 for details). A key attribute of our approach is the flexibility in incorporating any a posteriori elliptic error estimators available, as the reconstruction-type approach, allows to separate the challenges in the a posteriori error estimation of elliptic and time-evolution errors. To facilitate a wide range of applications, we will present the theory within a Gelfand-triple-type abstract setting allowing, for instance, both second- and fourth-order spatial operators. This generality comes at the possible expense of different, yet quantitatively analogous, computable constants in the resulting a posteriori error estimators compared to the bounds in $[16,17,19]$. For instance, when the equilibrated flux elliptic error estimators from [3] are used (with $\mathscr{X}=\mathrm{H}_{0}^{1}(\Omega)$ and $\mathscr{H}=\mathrm{L}_{2}(\Omega)$ ), we recover similar estimators to the upper bounds derived in [16,17]. Importantly, however, the work [16] shows that these are also lower bounds for the "joint-norm" of $\mathrm{H}^{1}\left(I ; \mathscr{X}^{\prime}\right) \cap \mathrm{L}_{2}(I ; \mathscr{X})$, and the article [17] does the same for the $\mathrm{L}_{2}(I ; \mathscr{X})$ under the condition $h^{2}<c \tau$, relating the mesh-size $h$ with the time-step $\tau$ for some constant $c>0$. Also, in the present work, we are not concerned with the interesting question of convergence of adaptive algorithms as in [19]. Crucially, however, the novel space-time reconstruction, allows for the proof of an a posteriori error bound for the $\mathrm{L}_{\infty}(I ; \mathscr{H})$-norm, which appears to be of optimal order; this result, to the best of our reading, is not captured in $[16,17,19]$.

Outline. The remainder of this work is structured as follows. In Sect. 2 we set up the abstract framework for the paper by introducing the model parabolic PDE problem and its DG-in-time and conforming Galerkin spatial discretization. Furthermore, in Sect. 3 , we provide the necessary technical tools for the ensuing analysis, and state their essential properties. In Sect. 4, we derive a posteriori error bounds in the $\mathrm{L}_{2}(I ; \mathscr{X})$ norm using a time reconstruction and a novel elliptic reconstruction which includes mesh-change; this technical novelty is revealed to be crucial in our setting. In Sect. 5, upon defining a new space-time reconstruction, we derive a posteriori error bounds in the $\mathrm{L}_{\infty}(I ; \mathscr{H})$-norm. Finally, in Sect. 6 we briefly discuss an approach to arriving at $\mathrm{H}^{1}\left(I ; \mathscr{X}^{\prime}\right)$-type a posteriori error estimates.

\section{Model problem and space-time discretization}

We introduce most of the notation and technical background for the paper. In Sect. 2.1 we provide the functional analytic set-up for the abstract heat equation, a related 
concrete Example 2.2, and we present the fully discrete numerical scheme in Sect. 2.3 .

\subsection{Abstract setting}

Throughout this work, Bochner spaces will be used. To that end, given an interval $J \subset \mathbb{R}$, and a real Hilbert space $\mathscr{Z}$ with inner product $(\cdot, \cdot) \mathscr{Z}$ and induced norm $\|\cdot\|_{\mathscr{Z}}$, we define

$$
\|u\|_{\mathrm{L}_{p}(J ; \mathscr{Z})}= \begin{cases}\left(\int_{J}\|u(t)\|_{\mathscr{Z}}^{p} \mathrm{~d} t\right)^{1 / p}, & 1 \leq p<\infty \\ {\operatorname{ess} \sup _{t \in J}\|u(t)\|_{\mathscr{Z}},} \quad p=\infty .\end{cases}
$$

We write $\mathrm{L}_{p}(J ; \mathscr{Z})$ to signify the space of measurable functions $u: J \rightarrow \mathscr{Z}$ such that the corresponding norm is bounded. Note that $\mathrm{L}_{2}(J ; \mathscr{Z})$ is a Hilbert space with inner product and induced norm given by

$$
(u, v)_{\mathrm{L}_{2}(J ; \mathscr{Z})}=\int_{J}(u(t), v(t)) \mathscr{Z} \mathrm{d} t,
$$

and $\|u\|_{\mathrm{L}_{2}(J ; \mathscr{Z})}:=(u, u)_{\mathrm{L}_{2}(J ; \mathscr{Z})}^{1 / 2}$, respectively. We also let $\mathrm{H}^{1}(J ; \mathscr{Z})$ be the Sobolev space of all functions in $\mathrm{L}_{2}(J ; \mathscr{Z})$ whose (weak temporal) derivative is bounded in $\mathrm{L}_{2}(J ; \mathscr{Z})$, with the norm

$$
\|u\|_{\mathrm{H}^{1}(J ; \mathscr{Z})}=\left(\int_{J}\left[\|u(t)\|_{\mathscr{Z}}^{2}+\left\|u^{\prime}(t)\right\|_{\mathscr{Z}}^{2}\right] \mathrm{d} t\right)^{1 / 2} .
$$

Finally, the space $\mathrm{C}^{0}(\bar{J} ; V)$ consists of all functions that are continuous on $\bar{J}$, the closure of $J$, with values in $\mathscr{Z}$, endowed with the standard maximum norm

$$
\|u\|_{\mathrm{C}^{0}(\bar{J} ; \mathscr{Z})}=\max _{t \in \bar{J}}\|u(t)\|_{\mathscr{Z}}
$$

We consider henceforth two (real) Hilbert spaces $\mathscr{X}$ and $\mathscr{H}$ forming a Gelfand triple

$$
\mathscr{X} \hookrightarrow \mathscr{H} \hookrightarrow \mathscr{X}^{\prime}
$$

where $\mathscr{X}^{\prime}$ denotes the dual of $\mathscr{X}$. The duality pairing $\langle\cdot \mid \cdot\rangle$ of $\mathscr{X}^{\prime}$ and $\mathscr{X}$ can be seen as a continuous extension of the inner product $(\cdot, \cdot) \mathscr{H}$. In particular, identifying $\mathscr{H}^{\prime} \simeq$ $\mathscr{H}$, for $u \in \mathscr{H}$ and $v \in \mathscr{X}$, there holds

$$
\langle u \mid v\rangle=(u, v)_{\mathscr{H}}
$$

see, e.g., [32, §7.2] for details.

Moreover, let

$$
\mathscr{A}: \mathscr{X} \rightarrow \mathscr{X}^{\prime}
$$


be a self-adjoint linear elliptic operator continuous and coercive in the sense that there exist constants $\beta \geq \alpha>0$ such that

$$
\begin{aligned}
& \langle\mathscr{A} v \mid w\rangle \leq \beta\|v\|_{\mathscr{X}}\|w\|_{\mathscr{X}} \text { for each } v, w \in \mathscr{X}, \\
& \langle\mathscr{A} v \mid v\rangle \geq \alpha\|v\|_{\mathscr{X}}^{2} \text { for each } v \in \mathscr{X} .
\end{aligned}
$$

Given an initial value $u_{0} \in \mathscr{H}$, a final time $T>0$, denoting henceforth the time interval

$$
I:=(0, T]
$$

and given a source function $f \in \mathrm{L}_{2}\left(I ; \mathscr{X}^{\prime}\right)$, we are interested in a Galerkin-type numerical approximation of the function

$$
u \in \mathrm{H}^{1}\left(I ; \mathscr{X}^{\prime}\right) \cap \mathrm{L}_{2}(I ; \mathscr{X}),
$$

which solves uniquely the linear parabolic initial value problem

$$
u^{\prime}+\mathscr{A} u=f \text { and } u(0)=u_{0} .
$$

Incidentally, due to the continuous embedding

$$
\mathrm{H}^{1}\left(I ; \mathscr{X}^{\prime}\right) \cap \mathrm{L}_{2}(I ; \mathscr{X}) \hookrightarrow \mathrm{C}^{0}(\bar{I} ; \mathscr{H}),
$$

it follows that $u$ belongs to $\mathrm{C}^{0}(\bar{I} ; \mathscr{H})$ [32, e.g., Lemma 7.3] and the initial condition in (2.11) makes sense.

2.2 Example (Concrete elliptic operators) A commonly encountered situation which can be cast in the above framework is the classical linear diffusion equation, i.e. $\mathscr{A} v=-\nabla \cdot[A \nabla v]$, where, for a given open, connected, and bounded domain $\Omega \subset \mathbb{R}^{d}$, $d=1,2$ or 3 , we consider a given symmetric matrix-valued function $\boldsymbol{A}: \Omega \rightarrow \mathbb{R}^{d \times d}$, $\boldsymbol{A} \in \mathrm{L}_{\infty}(\Omega)^{d \times d}$, satisfying

$$
\boldsymbol{v}^{\top} \boldsymbol{A}(\boldsymbol{x}) \boldsymbol{v} \geq \alpha|\boldsymbol{v}|^{2} \text { for each } \boldsymbol{x} \in \Omega, \text { for each } \boldsymbol{v} \in \mathbb{R}^{d},
$$

for some constant $\alpha>0$. Here, we choose, e.g., $\mathscr{H}:=\mathrm{L}_{2}(\Omega), \mathscr{X}:=\mathrm{H}_{0}^{1}(\Omega)$, and $\mathscr{X}^{\prime}:=\mathrm{H}^{-1}(\Omega)$ to be the typical function spaces in the context of second-order linear elliptic PDEs with homogeneous Dirichlet boundary condition $u=0$ on $\partial \Omega$.

Another possible choice, e.g., is $\mathscr{H}:=\mathrm{L}_{2}(\Omega), \mathscr{X}:=\mathrm{H}_{0}^{2}(\Omega)$, and $\mathscr{X}^{\prime}:=$ $\mathrm{H}^{-2}(\Omega)$, for the case of a fourth-order parabolic problem with essential boundary conditions. 


\subsection{Time discontinuous and space conforming Galerkin approximation}

Given a (real) linear space $\mathscr{Z}$, the space of all $\mathscr{Z}$-valued polynomials of degree at most $r$, with $r \in \mathbb{N}_{0}$, on $\mathbb{R}$ is defined by

$$
\mathbb{P}^{r}(\mathscr{Z}):=\left\{p: \mathbb{R} \rightarrow \mathscr{Z}: p(x)=\sum_{i=0}^{r} z_{i} x^{i} \text { for some }\left(z_{0}, \ldots, z_{r}\right) \in \mathscr{Z}^{r+1}\right\}
$$

In addition, if $D \subseteq \mathbb{R}$, we define

$$
\mathbb{P}^{r}(D ; \mathscr{Z}):=\left\{\left.p\right|_{D}: p \in \mathbb{P}^{r}(\mathscr{Z})\right\}
$$

In order to introduce the discontinuous Galerkin time stepping scheme for (2.11), we consider a finite sequence of time nodes and time steps,

$$
0=t_{0}<t_{1}<\cdots<t_{N}=T \text {, and } \tau_{n}:=t_{n}-t_{n-1} \text { for } n=1, \ldots, N \text {, }
$$

as well as the corresponding time intervals

$$
I_{n}:= \begin{cases}\{0\} & \text { for } n=0 \\ \left(t_{n-1}, t_{n}\right] & \text { for } n=1, \ldots, N\end{cases}
$$

Thus, we have a partition $\mathscr{I}:=\left\{I_{n}: n=1, \ldots, N\right\}$ of the time interval $I$ given in (2.9).

Given a $\mathscr{I}$-piecewise continuous function $g: I \subseteq \mathbb{R} \rightarrow \mathscr{Z}$, we define its time jump across $t_{n}, n=0, \ldots, N-1$, for given $g\left(t_{0}^{-}\right)$, by

$$
\llbracket g \rrbracket_{n}:=g\left(t_{n}^{+}\right)-g\left(t_{n}^{-}\right)
$$

where we introduce the one-sided limits $g\left(t_{n}^{ \pm}\right)=\lim _{\epsilon \rightarrow 0^{+}} g\left(t_{n} \pm \epsilon\right)$. Moreover, we associate with the finite sequence of time instants $t_{0}, \ldots, t_{N}$ a finite sequence of finitedimensional conforming subspaces

$$
\mathbb{X}_{n} \subset \mathscr{X}, \text { for } n=0, \ldots, N
$$

For a generic $\mathscr{X}$-conforming Galerkin space $\mathbb{X}$, we signify by $\pi_{\mathbb{X}}$ the $\mathscr{H}$ orthogonal projection from $\mathscr{X}^{\prime}$ onto $\mathbb{X}$ :

$$
\begin{aligned}
\pi_{\mathbb{X}}: \mathscr{X}^{\prime} & \rightarrow \mathbb{X} \\
v & \mapsto \pi_{\mathbb{X}} v:\left(\pi_{\mathbb{X}} v, \mathrm{w}\right)_{\mathscr{H}}=\langle v \mid w\rangle \text { for each } \mathrm{w} \in \mathbb{X} .
\end{aligned}
$$

Note that, due to (2.6), for $v \in \mathscr{H}$, we have

$$
\left(\pi_{\mathbb{X}} v, \mathrm{w}\right)_{\mathscr{H}}=\langle v \mid \mathrm{w}\rangle=(v, \mathrm{w})_{\mathscr{H}} \text { for each } \mathrm{w} \in \mathbb{X}
$$

When $\mathbb{X}$ is $\mathbb{X}_{n}$, for some $n=0, \ldots, N$, we write $\pi_{n}$ to indicate $\pi_{\mathbb{X}}$. 
In order to introduce the time semidiscrete and space-time fully discrete spaces, let $r_{n} \in \mathbb{N}_{0}, n=1, \ldots, N$, be a polynomial degree. Then, consider the time semidiscrete Galerkin space

$$
\mathscr{Y}:=\left\{\mathrm{V}:(0, T] \rightarrow \mathscr{X}:\left.\mathrm{V}\right|_{I_{n}} \in \mathbb{P}^{r_{n}}\left(I_{n} ; \mathscr{X}\right) \text { for each } n=1, \ldots, N\right\},
$$

respectively, the space-time fully discrete Galerkin space

$$
\mathbb{Y}:=\left\{\mathrm{V}:(0, T] \rightarrow \mathscr{X}:\left.\mathrm{V}\right|_{I_{n}} \in \mathbb{P}^{r_{n}}\left(I_{n} ; \mathbb{X}_{n}\right) \text { for each } n=1, \ldots, N\right\},
$$

where $\mathbb{P}^{r_{n}}\left(I_{n} ; \mathbb{X}_{n}\right)$ are the space-time Galerkin subspaces. The fully discrete timediscontinuous Galerkin and spatially-conforming approximation of (2.11) is then an $\mathscr{I}$-piecewise continuous function $\mathrm{U} \in \mathbb{Y}$, such that

$$
\mathrm{U}\left(t_{0}^{-}\right):=\pi_{0} u_{0}
$$

and for $n=1, \ldots, N$,

$$
\int_{I_{n}}\left[\left(\mathrm{U}^{\prime}, \mathrm{V}\right)_{\mathscr{H}}+\langle\mathscr{A} \mathrm{U} \mid \mathrm{V}\rangle\right] \mathrm{d} t+\left(\llbracket \mathrm{U} \rrbracket_{n-1}, \mathrm{~V}\left(t_{n-1}^{+}\right)\right)_{\mathscr{H}}=\int_{I_{n}}\langle f \mid \mathrm{V}\rangle \mathrm{d} t
$$

for each $\mathrm{V} \in \mathbb{P}^{r_{n}}\left(I_{n} ; \mathbb{X}_{n}\right)$, where $\llbracket \mathrm{U} \rrbracket_{0}=\mathrm{U}\left(t_{0}^{+}\right)-\pi_{0} u_{0}$

\section{Reconstructions}

We will next introduce some technical essentials. The main tools are the time lifting (Sect. 3.1), the time reconstruction (Sect. 3.3), and a new variant of the elliptic reconstruction from [23] for fully discrete schemes (Sect. 3.6). In Sect. 3.7 we postulate the availability of a posteriori error estimators for elliptic residuals, and we give some pointers to the relevant literature. In addition, we discuss various error estimates that measure the time reconstruction error; in particular, we state two identities which follow directly, respectively, from [35, Theorem 2] and, taking into account the explicit representation of the time reconstruction, from [20, Lemma 1].

\subsection{Time lifting}

Let us consider, for given $n=1, \ldots, N$, a linear time lifting operator

$$
\chi_{n}: \mathscr{H} \rightarrow \mathbb{P}^{r_{n}}\left(I_{n} ; \mathscr{H}\right)
$$

It is defined, for each $w \in \mathscr{H}$, by the Riesz representation

$$
\int_{I_{n}}\left(\chi_{n}(w), V\right)_{\mathscr{H}}=\left(w, V\left(t_{n-1}^{+}\right)\right)_{\mathscr{H}} \quad \text { for each } V \in \mathbb{P}^{r_{n}}\left(I_{n} ; \mathscr{H}\right) .
$$


3.2 Lemma (Space invariance under time lifting) For any linear subspace $\mathscr{W} \subseteq \mathscr{H}$, the time lifting from (3.1) and (3.2) satisfies

$$
w \in \mathscr{W} \Longrightarrow \chi_{n}(w) \in \mathbb{P}^{r_{n}}\left(I_{n} ; \mathscr{W}\right)
$$

In particular, writing $\mathbb{1}_{A}$ for the indicator function on a generic set A, and assuming $\mathrm{w}_{n} \in \mathbb{X}_{n}$ for each $n=1, \ldots, N$, we have

$$
\sum_{n=1}^{N} \chi_{n}\left(\mathrm{w}_{n}\right) \mathbb{1}_{I_{n}} \in \mathbb{Y}
$$

Proof This result is a straightforward consequence of the explicit representation of $\chi_{n}$ as described in [35, Lemma 6].

\subsection{Time reconstruction}

Let us define the time-reconstruction $\widehat{W}$ of a given time-discrete function

$$
W \in\left\{\mathrm{V}:(0, T] \rightarrow \mathscr{H}:\left.\mathrm{V}\right|_{I_{n}} \in \mathbb{P}^{r_{n}}\left(I_{n} ; \mathscr{H}\right) \text { for each } n=1, \ldots, N\right\}
$$

as follows: for each $n=1, \ldots, N$, we let $\left.\widehat{W}\right|_{I_{n}} \in \mathbb{P}^{r_{n}+1}\left(I_{n} ; \mathscr{H}\right)$ satisfy

$$
\left.\widehat{W}\right|_{I_{n}}(t):=W\left(t_{n-1}^{-}\right)+\int_{t_{n-1}}^{t}\left[W^{\prime}(s)+\chi_{n}\left(\llbracket W \rrbracket_{n-1}\right)(s)\right] \mathrm{d} s \quad \text { for } t \in \bar{I}_{n} .
$$

Equivalently, we note the following characterization of $\widehat{W}$ in weak form on each $I_{n}$,

$$
\int_{I_{n}}\left(\widehat{W}^{\prime}, V\right)_{\mathscr{H}} \mathrm{d} t=\left(\llbracket W \rrbracket n-1, V\left(t_{n-1}^{+}\right)\right)_{\mathscr{H}}+\int_{I_{n}}\left(W^{\prime}, V\right)_{\mathscr{H}} \mathrm{d} t,
$$

for each $V \in \mathbb{P}^{r_{n}}\left(I_{n} ; \mathscr{H}\right)$, with the initial condition

$$
\widehat{W}\left(t_{n-1}^{+}\right):=W\left(t_{n-1}^{-}\right),
$$

for $n=1, \ldots, N$; cf. [27,35]. Evidently, the above construction carries over to any linear subspace $\mathscr{W} \subset \mathscr{H}$ in an obvious way.

3.4 Proposition (Time-reconstruction error identities) Consider any (real) Hilbert space $\mathscr{W}$, and $\left.W\right|_{I_{n}} \in \mathbb{P}^{r_{n}}\left(I_{n} ; \mathscr{W}\right), n=1, \ldots, N$, with $\widehat{W}$ defined from $W$ through (3.6). Then, for given $W\left(t_{0}^{-}\right) \in \mathscr{W}$, the following approximation identities hold

$$
\|W-\widehat{W}\|_{\mathrm{L}_{2}\left(I_{n} ; \mathscr{W}\right)}=C_{\tau_{n}, r_{n}}\left\|\llbracket W \rrbracket_{n-1}\right\| \mathscr{W},
$$

where

$$
C_{\tau, r}:=\left(\frac{\tau(r+1)}{(2 r+1)(2 r+3)}\right)^{1 / 2}
$$


and

$$
\|W-\widehat{W}\|_{\mathrm{L}_{\infty}\left(I_{n} ; \mathscr{W}\right)}=\left\|\llbracket W \rrbracket_{n-1}\right\|_{\mathscr{W}}
$$

Proof The identity (3.9) was first proved in [27, Lemma 2.2], and extended to this exact form in [35, Theorem 2] accounting for the dependence on the polynomial degree explicitly. The second equality (3.11) follows directly by combining the explicit representation formula derived in [35, Eq. (33)] with [20, Lemma 1].

3.5 Remark (Continuity of the time-reconstruction) Owing to [28, Lemma 2.1], the semidiscrete (spatially exact) time-reconstruction (3.6) originally defined in [28] and [35] is a continuous function in time. In particular, the time-reconstruction $\widehat{U}$ of the fully discrete solution $U$, defined in (2.24) and (2.25), is still continuous across the time nodes $t_{0}, \ldots, t_{N-1}$, despite having $\pi_{n} \widehat{U} \neq \widehat{U}$, on $I_{n}$, when the spatial mesh changes across $t_{n-1}$ in a non-hierarchical fashion.

\subsection{Elliptic reconstruction}

Let $\mathbb{X} \subset \mathscr{X}$ be a generic conforming Galerkin space. Then, given the elliptic operator $\mathscr{A}$ from (2.7), we define the discrete elliptic operator $A_{\mathbb{X}}: \mathbb{X} \rightarrow \mathbb{X}$, for each $\mathrm{w} \in \mathbb{X}$, as $\mathrm{A}_{\mathbb{X}} \mathrm{w} \in \mathbb{X}$ such that

$$
\left(\mathrm{A}_{\mathbb{X}} \mathrm{w}, \mathrm{v}\right)_{\mathscr{H}}=\langle\mathscr{A} \mathrm{w} \mid \mathrm{v}\rangle \text { for each } \mathrm{v} \in \mathbb{X}
$$

From the ellipticity of $\mathscr{A}$, it follows that $\mathrm{A}_{\mathbb{X}}: \mathbb{X} \rightarrow \mathbb{X}$ is invertible. Note that the discrete elliptic operator's domain may be extended from $\mathbb{X}$ to all of $\mathscr{X}$; indeed, this may be convenient in some cases where we are ready to give up its invertibility. If $\mathbb{X}$ is one of $\mathbb{X}_{n}$ s, for some $n=0, \ldots, N$, we denote $A_{\mathbb{X}_{n}}$ by $A_{n}$.

To optimize on the structure of the mesh-change indicator below, we use a nonstandard elliptic reconstruction on each time interval $I_{n}$. To that end, for each $t \in I_{n}$, we define the elliptic reconstruction $\widetilde{U}(t) \in \mathscr{X}$, by

$$
\langle\mathscr{A} \widetilde{U}(t) \mid v\rangle=\left(A_{n} \mathrm{U}(t)+\pi_{n} \widehat{\mathrm{U}}^{\prime}(t)-\widehat{U}^{\prime}(t), v\right)_{\mathscr{H}} \quad \text { for each } v \in \mathscr{X}
$$

where $\widehat{U}$ is the time-reconstruction of the fully discrete solution $U$ from (2.24) and (2.25). It follows that $\widetilde{U} \in \mathscr{Y}$ and may be written implicitly, for any $n=1, \ldots, N$, as the solution of the $t$-dependent elliptic problem

$$
\mathscr{A} \widetilde{U}(t)=A_{n} \mathrm{U}(t)+\pi_{n} \widehat{\mathrm{U}}^{\prime}(t)-\widehat{U}^{\prime}(t) \quad \text { for } t \in I_{n} \text {. }
$$

The initial value of $\widetilde{U}$ is given by

$$
\tilde{U}(0)=\tilde{U}\left(t_{0}^{-}\right):=\pi_{0} u\left(t_{0}^{-}\right)=\pi_{0} u_{0},
$$

with $u_{0} \in \mathscr{H}$ from (2.11). 
Upon restricting the test functions in (3.13) to $\mathbb{X}_{n}$, we obtain

$$
\langle\mathscr{A} \tilde{U}(t) \mid \mathrm{v}\rangle=\left(A_{n} \mathrm{U}(t), \mathrm{v}\right)_{\mathscr{H}}=\langle\mathscr{A} \mathrm{U}(t) \mid \mathrm{v}\rangle \quad \text { for each } \mathrm{v} \in \mathbb{X}_{n},
$$

cf. (3.12). Evidently this identity implies

$$
\int_{I_{n}}\langle\mathscr{A} \tilde{U} \mid \mathrm{V}\rangle \mathrm{d} t=\int_{I_{n}}\left(A_{n} \mathrm{U}, \mathrm{V}\right)_{\mathscr{H}} \mathrm{d} t
$$

for each $\vee \in \mathbb{P}^{r_{n}}\left(I_{n} ; \mathbb{X}_{n}\right), n=1, \ldots, N$.

\subsection{Assumption (elliptic a posteriori error estimates)}

For given $g \in \mathscr{H}$, consider the abstract elliptic problem of finding $w \in \mathscr{X}$ such that $\mathscr{A} w=g$. Moreover, let $\mathbb{X} \subseteq \mathscr{X}$ be a generic $\mathscr{X}$-conforming Galerkin space, and let $w \in \mathbb{X}$ be $w$ 's Galerkin approximation in $\mathbb{X}$, defined implicitly as the solution of $\mathrm{A}_{\mathbb{X}} \mathrm{W}=\pi_{\mathbb{X}} g$. Then some a posteriori error bound holds, viz.,

$$
\|w-\mathrm{w}\|_{\mathscr{Z}} \leq \mathscr{E}_{\mathscr{Z}, \mathbb{X}}[\mathrm{w}, g]
$$

with a suitable a posteriori error estimator $\mathscr{E}_{\mathscr{Z}, \mathbb{X}}$, which we assume to be available for $\mathscr{Z}$ representing any of the spaces $\mathscr{X}, \mathscr{H}$ or $\mathscr{X}^{\prime}$. Recalling (3.14), assumption (3.18) allows, for instance, to get a posteriori error control of the elliptic reconstruction error in the $\mathscr{Z}$-norm, i.e.

$$
\|\widetilde{U}-\mathrm{U}\|_{\mathscr{Z}} \leq \mathscr{E}_{\mathscr{Z}, \mathbb{X}_{n}}\left[\mathrm{U}, A_{n} \mathrm{U}+\pi_{n} \widehat{U}^{\prime}-\widehat{U}^{\prime}\right] \text { on } I_{n},
$$

for the selection of spaces $\mathscr{Z}=\mathscr{X}, \mathscr{H}$, or $\mathscr{X}^{\prime}$. Details on such a posteriori error estimates can be found, e.g., in [2,4,5,9]. It is worth mentioning that $w$ does not need to belong to $\mathbb{X}$ for (3.18) to hold; instead, it is usually enough that $(w-\mathrm{w})$ is $\mathscr{A}$-orthogonal to $\mathbb{X}$ in order to derive elliptic a posteriori error estimates.

\subsection{Pointwise form}

For $n=1, \ldots, N$, we denote by

$$
\Pi_{n}: \mathrm{L}_{2}\left(I_{n} ; \mathscr{X}^{\prime}\right) \rightarrow \mathbb{P}^{r_{n}}\left(I_{n} ; \mathbb{X}_{n}\right), \quad f \mapsto \Pi_{n} f,
$$

the time-local fully discrete $\mathrm{L}_{2}\left(I_{n} ; \mathscr{H}\right)$-orthogonal projection defined by

$$
\int_{I_{n}}\left(\Pi_{n} f, \mathrm{~V}\right)_{\mathscr{H}} \mathrm{d} t=\int_{I_{n}}\langle f \mid \mathrm{V}\rangle \mathrm{d} t \text { for each } \mathrm{V} \in \mathbb{P}^{r_{n}}\left(I_{n} ; \mathbb{X}_{n}\right)
$$

and its time-global counterpart

$$
\begin{aligned}
\Pi: \mathrm{L}_{2}\left(I ; \mathscr{X}^{\prime}\right) & \rightarrow \mathbb{Y} \\
f & \mapsto \Pi f:\left.\Pi f\right|_{I_{n}}=\Pi_{n} f \text { for each } n=1, \ldots, N .
\end{aligned}
$$


Let $\mathrm{V} \in \mathbb{Y}$, and note that using Definition 3.3 and identity (3.17), the fully discrete local DG formulation (2.25) transforms into

$$
\int_{I_{n}}\left(\widehat{\mathrm{U}}^{\prime}+A_{n} \mathrm{U}-f, \mathrm{~V}\right)_{\mathscr{H}} \mathrm{d} t=0, \quad n=1, \ldots, N
$$

Thus, for each $n=1, \ldots, N$, we have

$$
\pi_{n} \widehat{\mathrm{U}}^{\prime}(t)+A_{n} \mathrm{U}(t)-\Pi_{n} f(t)=0 \quad \text { for each } t \in I_{n},
$$

noting that $\Pi_{n} \widehat{U}^{\prime}=\pi_{n} \widehat{U}^{\prime}$ from Fubini's Theorem. Hence, from the elliptic reconstruction (3.13), we deduce the pointwise form

$$
\widehat{U}^{\prime}(t)+\mathscr{A} \widetilde{U}(t)=\Pi_{n} f(t) \text { for each } t \in I_{n} .
$$

3.9 Remark Upon noting the trivial identity for $t \in I_{n}$,

$$
\mathscr{A} \tilde{U}(t)=\Pi_{n} f(t)-\widehat{U}^{\prime}(t)
$$

we observe that the above definition of the elliptic reconstruction constitutes a high order extension of the zero-th order version from [6, Definition 6.1].

\section{$4 \mathrm{~L}_{2}(I ; \mathscr{X})$-norm a posteriori error analysis}

To highlight the potential generality of the reconstruction approach, we first embark on proving a posteriori error bounds in the $\mathrm{L}_{2}(I ; \mathscr{X})$-norm. As we will see below, the resulting bounds are qualitatively closely related to the bounds from $[16,17,19]$ when $\mathscr{X}=\mathrm{H}^{1}(\Omega)$. A key attribute of the approach presented below is that the resulting a posteriori bounds are flexible with respect to the choice of respective elliptic bounds, such as residual, recovery, or flux-reconstruction ones.

\subsection{Errors}

Introduce the following errors

$$
\widehat{\sigma}:=\widehat{U}-U, \quad \widetilde{\sigma}:=\widetilde{U}-U
$$

and the corresponding remainder error

$$
\widehat{e}:=u-\widehat{U} \text { and } \widetilde{e}:=u-\widetilde{U}
$$

whereby the full error can be decomposed as

$$
e:=u-\mathrm{U}=\widehat{e}+\widehat{\sigma}=\widetilde{\sigma}+\widetilde{e} .
$$


Recalling that our ultimate goal is to find a posteriori estimates for $e$, and noting that such estimates for $\widehat{\sigma}$ and $\widetilde{\sigma}$ are provided by Proposition 3.4 and Assumption 3.7, respectively, it will suffice to estimate $\widehat{e}$, or $\widetilde{e}$, in terms of $\widehat{\sigma}$ or $\widetilde{\sigma}$.

4.2 Lemma (Time reconstruction error bounds) For $n=1, \ldots, N$, we have the explicitly computable bounds

$$
\|\widehat{\sigma}\|_{\mathrm{L}_{2}\left(0, t_{n} ; \mathscr{X}\right)}^{2}=\sum_{m=1}^{n} C_{\tau_{m}, r_{m}}^{2}\|\llbracket \mathrm{U} \rrbracket m-1\|_{\mathscr{X}}^{2}
$$

and

$$
\|\widehat{\sigma}\|_{\mathrm{L}_{\infty}\left(0, t_{n} ; \mathscr{H}\right)}^{2}=\max _{m=1, \ldots, n}\|\llbracket \mathrm{U} \rrbracket m-1\|_{\mathscr{H}}^{2}
$$

Proof The proof follows immediately from Proposition 3.4.

4.3 Lemma (Space reconstruction error bounds) Assume the availability of elliptic a posteriori error bounds as per Assumption 3.7. Then, for $n=1, \ldots, N$, we have the explicitly computable bound

$$
\|\widetilde{\sigma}\|_{\mathrm{L}_{2}\left(0, t_{n} ; \mathscr{X}\right)}^{2} \leq \sum_{m=1}^{n} \int_{I_{m}} \mathscr{E} \mathscr{X}, \mathbb{X}_{n}\left[\mathrm{U}, A_{n} \mathrm{U}+\pi_{n} \widehat{U}^{\prime}-\widehat{U}^{\prime}\right]^{2} \mathrm{~d} t
$$

We can now prove the first main result of this work.

4.4 Theorem $\left(\mathrm{L}_{2}(I ; \mathscr{X})\right.$-norm a posteriori error bound) Let Assumption 3.7 hold. Then, for $n=1, \ldots, N$, and

$$
\left(1+\frac{\alpha}{\beta}\right)^{-1} \leq \vartheta^{2} \leq 1+\frac{\alpha}{\beta}
$$

we have the following a posteriori error bound

$$
\begin{aligned}
\sqrt{\alpha}\|e\|_{\mathrm{L}_{2}\left(0, t_{n} ; \mathscr{X}\right) \leq} & \eta^{\mathrm{in}}+c_{\alpha, \beta} \vartheta^{-1} \eta_{n}^{\mathrm{osc}}+c_{\alpha, \beta} \vartheta \beta\left(\sum_{m=1}^{n} C_{\tau_{m}, r_{m}}^{2}\left\|\llbracket \mathrm{U} \rrbracket_{m-1}\right\|_{\mathscr{X}}^{2}\right)^{1 / 2} \\
& \left.+\left(\sqrt{\alpha}+c_{\alpha, \beta} \vartheta \beta\right)\left(\sum_{m=1}^{n} \int_{I_{m}} \mathscr{E} \mathscr{X}, \mathbb{X}_{n}\left[\mathrm{U}, A_{n} \mathrm{U}+\pi_{n} \widehat{\mathrm{U}}^{\prime}-\widehat{\mathrm{U}}^{\prime}\right]^{2} \mathrm{~d} t\right)\right)^{1 / 2},
\end{aligned}
$$

where $c_{\alpha, \beta}:=\sqrt{\alpha^{-1}+\beta^{-1}}$, with the constants $\alpha, \beta$ from (2.8), and $\eta_{n}^{\text {osc }}:=$ $\|\Pi f-f\|_{\mathrm{L}_{2}\left(0, t_{n} ; \mathscr{X}^{\prime}\right)}$, and $\eta^{\text {in }}:=\left\|u_{0}-\pi_{0} u_{0}\right\|_{\mathscr{H}}$ to signify the data oscillation and initial error indicators, respectively.

Proof From (3.25) and the PDE (2.11), on each $I_{n}$, we have

$$
\widehat{e}^{\prime}+\mathscr{A} \widetilde{e}=u^{\prime}+\mathscr{A} u-\widehat{U}^{\prime}-\mathscr{A} \tilde{U}=f-\Pi_{n} f .
$$


Testing (4.9) with $\widehat{e}$, we deduce

$$
\left(\widehat{e}^{\prime}, \widehat{e}\right)_{\mathscr{H}}+\langle\mathscr{A} \widetilde{e} \mid \widetilde{e}\rangle=\left\langle f-\Pi_{n} f \mid \widehat{e}\right\rangle+\langle\mathscr{A} \widetilde{e} \mid \widehat{\sigma}-\widetilde{\sigma}\rangle
$$

Note that the term $\Pi_{n} f-f$ occurring in the above estimator is computable signifying the so-called oscillation error. Thanks to the continuity of $\widehat{e}$, we can integrate the last identity on $\left(0, t_{n}\right)$, for some $n=1, \ldots, N$, and through standard arguments, we obtain

$$
\begin{aligned}
\frac{1}{2}\left\|\widehat{e}\left(t_{n}\right)\right\|_{\mathscr{H}}^{2}+\alpha\|\widetilde{e}\|_{\mathrm{L}_{2}\left(0, t_{n} ; \mathscr{X}\right) \leq}^{2} \leq & \frac{1}{2}\|\widehat{e}(0)\|_{\mathscr{H}}^{2}+\beta\|\widetilde{e}\|_{\mathrm{L}_{2}\left(0, t_{n} ; \mathscr{X}\right)}\|\widehat{\sigma}-\widetilde{\sigma}\|_{\mathrm{L}_{2}\left(0, t_{n} ; \mathscr{X}\right)} \\
& +\eta_{n}^{\text {osc }}\left(\|\widetilde{e}\|_{\mathrm{L}_{2}\left(0, t_{n} ; \mathscr{X}\right)}+\|\widehat{\sigma}-\widetilde{\sigma}\|_{\mathrm{L}_{2}\left(0, t_{n} ; \mathscr{X}\right)}\right)
\end{aligned}
$$

using the continuity and coercivity of $\mathscr{A}$, cf. (2.8). For any $\vartheta>0$ observe the identity

$$
\begin{aligned}
\beta\|\widetilde{e}\|_{\mathrm{L}_{2}\left(0, t_{n} ; \mathscr{X}\right)}\|\widehat{\sigma}-\widetilde{\sigma}\|_{\mathrm{L}_{2}\left(0, t_{n} ; \mathscr{X}\right)}+\eta_{n}^{\text {osc }}\left(\|\widetilde{e}\|_{\mathrm{L}_{2}\left(0, t_{n} ; \mathscr{X}\right)}+\|\widehat{\sigma}-\widetilde{\sigma}\|_{\mathrm{L}_{2}\left(0, t_{n} ; \mathscr{X}\right)}\right) \\
=\frac{\alpha}{2}\|\widetilde{e}\|_{\mathrm{L}_{2}\left(0, t_{n} ; \mathscr{X}\right)}^{2}+\frac{1}{2}\left(\alpha^{-1}+\beta^{-1}\right)\left(\vartheta \beta\|\widehat{\sigma}-\widetilde{\sigma}\|_{\mathrm{L}_{2}\left(0, t_{n} ; \mathscr{X}\right)}+\vartheta^{-1} \eta_{n}^{\mathrm{osc}}\right)^{2} \\
\quad-\frac{1}{2 \alpha}\left(\beta\|\widehat{\sigma}-\widetilde{\sigma}\|_{\mathrm{L}_{2}\left(0, t_{n} ; \mathscr{X}\right)}-\alpha\|\widetilde{e}\|_{\mathrm{L}_{2}\left(0, t_{n} ; \mathscr{X}\right)}+\eta_{n}^{\text {osc }}\right)^{2} \\
\quad+\frac{1}{2}\left(\alpha^{-1}-\vartheta^{2}\left(\alpha^{-1}+\beta^{-1}\right)\right) \beta^{2}\|\widehat{\sigma}-\widetilde{\sigma}\|_{\mathrm{L}_{2}\left(0, t_{n} ; \mathscr{X}\right)}^{2} \\
+\frac{1}{2}\left(\alpha^{-1}-\vartheta^{-2}\left(\alpha^{-1}+\beta^{-1}\right)\right)\left(\eta_{n}^{\text {osc }}\right)^{2} .
\end{aligned}
$$

Notice that the last two terms are non-positive for $\vartheta$ as in (4.7). Then, from (4.11), we infer

$$
\alpha\|\widetilde{e}\|_{\mathrm{L}_{2}\left(0, t_{n} ; \mathscr{X}\right)}^{2} \leq\|\widehat{e}(0)\|_{\mathscr{H}}^{2}+\left(\alpha^{-1}+\beta^{-1}\right)\left(\vartheta \beta\|\widehat{\sigma}-\widetilde{\sigma}\|_{\mathrm{L}_{2}\left(0, t_{n} ; \mathscr{X}\right)}+\vartheta^{-1} \eta_{n}^{\mathrm{osc}}\right)^{2} .
$$

Taking square root and using the triangle inequality, $\|e\|_{\mathrm{L}_{2}\left(0, t_{n} ; \mathscr{X}\right)} \leq\|\widetilde{e}\|_{\mathrm{L}_{2}\left(0, t_{n} ; \mathscr{X}\right)}+$ $\|\widetilde{\sigma}\|_{\mathrm{L}_{2}\left(0, t_{n} ; \mathscr{X}\right)}$, we deduce

$$
\begin{aligned}
\sqrt{\alpha}\|e\|_{\mathrm{L}_{2}\left(0, t_{n} ; \mathscr{X}\right) \leq} & \|\widehat{e}(0)\| \mathscr{H}+c_{\alpha, \beta} \vartheta \beta\|\widehat{\sigma}\|_{\mathrm{L}_{2}\left(0, t_{n} ; \mathscr{X}\right)} \\
& +\left(\sqrt{\alpha}+c_{\alpha, \beta} \vartheta \beta\right)\|\widetilde{\sigma}\|_{\mathrm{L}_{2}\left(0, t_{n} ; \mathscr{X}\right)}+c_{\alpha, \beta} \vartheta^{-1} \eta_{n}^{\text {osc }} .
\end{aligned}
$$

Invoking now Lemmas 4.2 and 4.3 , and noting that $\|\widehat{e}(0)\|_{\mathscr{H}}=\left\|u_{0}-\pi_{0} u_{0}\right\|_{\mathscr{H}}$, the result already follows.

4.5 Remark For the particular case $\mathscr{A}=-\Delta, \mathscr{X}=\mathrm{H}_{0}^{1}(\Omega)$ and $\mathscr{H}=\mathrm{L}_{2}(\Omega)$, cf. Example 2.2, note that the constants in (2.8) can be chosen to be $\alpha=\beta=1$. Especially, selecting $\vartheta^{2}=1 / 2$, we have $c_{\alpha, \beta} \vartheta=1$ in (4.8).

4.6 Remark (Mesh change error via elliptic reconstruction) The elliptic reconstruction (3.13) features a mesh-change type term. This is an important departure from the (standard) elliptic reconstruction proposed in [24]; cf. also [6, Definition 6.1]. For 
instance, if $\mathscr{E} \mathscr{X}, \mathbb{X}_{n}$ from (3.18) is the standard residual energy norm a posteriori error estimator, the element residual includes the expression $\pi_{n} \widehat{U}^{\prime}-\widehat{U}^{\prime}$, which is effectively a mesh-change term. Indeed, from (3.6) we have on $I_{n}$ :

$$
\begin{aligned}
\pi_{n} \widehat{\mathrm{U}}^{\prime}-\widehat{\mathrm{U}}^{\prime} & =\pi_{n}\left(\mathrm{U}^{\prime}+\chi_{n}\left(\llbracket \mathrm{U} \rrbracket_{n-1}\right)\right)-\left(\mathrm{U}^{\prime}+\chi_{n}\left(\llbracket \mathrm{U} \rrbracket_{n-1}\right)\right) \\
& =\chi_{n}\left(\pi_{n} \llbracket \mathrm{U} \rrbracket_{n-1}-\llbracket \mathrm{U} \rrbracket_{n-1}\right)=\chi_{n}\left(\mathrm{U}\left(t_{n-1}^{-}\right)-\pi_{n} \mathrm{U}\left(t_{n-1}^{-}\right)\right),
\end{aligned}
$$

from the commutativity of the spatial projection $\pi_{n}$ and operations on the time variable. Let now $\varkappa_{n}: I_{n} \rightarrow \mathbb{R}$ be the polynomial of degree $r_{n}$ representing the time lifting $\chi_{n}$ from (3.2), i.e. such that

$$
\chi_{n}\left(\llbracket W \rrbracket_{n-1}\right)(t)=\varkappa_{n}(t) \llbracket W \rrbracket_{n-1},
$$

for all $t \in I_{n}$ and $W \in \mathscr{Y}$; we refer to [35, Lemmas $6 \&$ 7] or [20, Remark 1] for an explicit formula for $\varkappa_{n}$. Then, we can conclude

$$
\pi_{n} \widehat{\mathrm{U}}^{\prime}(t)-\widehat{\mathrm{U}}^{\prime}(t)=\varkappa_{n}(t)\left(\mathrm{U}\left(t_{n-1}^{-}\right)-\pi_{n} \mathrm{U}\left(t_{n-1}^{-}\right)\right), \quad t \in I_{n},
$$

i.e. the arbitrary order DG-analogue to the classical mesh-change indicator. We note that the representation (4.17) is particularly relevant in implementation as it can be used to efficiently realize the space reconstruction error bounds in Lemma 4.3. Finally, observing that $\varkappa_{n}$ takes its maximum at $t_{n-1}$, which follows from [35, Lemma 6], and recalling (3.2), it is possible to compute the maximum value of $\varkappa_{n}$ on $\bar{I}_{n}$ :

$$
\left\|\varkappa_{n}\right\|_{\mathrm{L}_{\infty}\left(I_{n}\right)}^{2}=\left|\varkappa_{n}\left(t_{n-1}\right)\right|^{2}=\int_{I_{n}} \varkappa_{n}(t)^{2} \mathrm{~d} t .
$$

Hence

$$
\left\|\varkappa_{n}\right\|_{\mathrm{L}_{\infty}\left(I_{n}\right)}=\left\|\varkappa_{n}\right\|_{\mathrm{L}_{2}\left(I_{n}\right)}=\frac{r_{n}+1}{\sqrt{\tau_{n}}},
$$

see [35, Proposition 2].

4.7 Remark (Reconstruction vs direct approach) As noted by one referee, it is possible to spare the use of elliptic reconstruction in the proof of the $\mathrm{L}_{2}(I ; \mathscr{X})$-norm a posteriori bound, for particular cases of operators $\mathscr{A}$ and of Gelfand triples (2.5), as discussed in $[16,17,19]$ for the specific case $\mathscr{A}=-\Delta, \mathscr{X}=\mathrm{H}_{0}^{1}(\Omega)$ and $\mathscr{H}=\mathrm{L}_{2}(\Omega)$; cf. Example 2.2. As a result, different a posteriori error estimators arise with, possibly, slightly different constants multiplying common terms in the estimator. Elliptic reconstruction, nonetheless, offers the ability to use various elliptic estimators from the literature in the bound: this feature may become important for multiscale operators $\mathscr{A}$, e.g., singular perturbations. In general, elliptic reconstruction allows for, crucial in some cases, flexibility in the handling for more complicated spatial operators $\mathscr{A}$, e.g., nonlinear or singularly perturbed operators $[6,7,18]$. 


\section{$5 \mathrm{~L}_{\infty}(I ; \mathscr{H})$-norm a posteriori error analysis}

We continue by proving an a posteriori error bound in the $\mathrm{L}_{\infty}(I ; \mathscr{H})$-norm, which appears to be of higher order than the $\mathrm{L}_{2}(I ; \mathscr{X})$-norm one presented above. A key difference with respect to the above proof of the $\mathrm{L}_{2}(I ; \mathscr{X})$-norm bound is the use of a combined space-time reconstruction defined below.

\subsection{Error-residual relation}

Set $w:=\widehat{\widetilde{U}}$, i.e. the time reconstruction of the elliptic reconstruction, noting that Definition 3.3 is still valid for functions on $\mathscr{X}$ in space. Now, introducing the time error

$$
\rho:=w-u,
$$

which is continuous on $\bar{I}=[0, T]$, and subtracting the PDE (2.11) from (3.25), we have:

$$
(\widehat{\mathrm{U}}-u)^{\prime}+\mathscr{A}(\widetilde{U}-u)=\Pi f-f,
$$

our aim being to deduce an evolution equation for $\rho$. To this end, we have

$$
\begin{aligned}
\rho^{\prime}+\mathscr{A} \rho & =\Pi f-f+(w-\widehat{U})^{\prime}+\mathscr{A}(w-\widetilde{U}) \\
& =\Pi f-f+(\widehat{\widetilde{U}-U})^{\prime}+\mathscr{A}(w-\widetilde{U})=: \xi,
\end{aligned}
$$

from the linearity of the time reconstruction.

We define $\mathcal{T}_{n} \wedge \mathcal{T}_{n-1}$ to be the finest common coarsening of the two meshes, with the associated largest common finite element subspace given by $\mathbb{X}_{n}^{\ominus}=\mathbb{X}_{n} \cap \mathbb{X}_{n-1}$, and a meshsize $h_{n}^{\ominus}=h_{n} \vee h_{n-1}$.

5.2 Lemma (Time reconstruction error bound) Let $t \in I_{n}, n=0,1 \ldots, N$. Then, we have the abstract estimate

$$
\|\mathscr{A}(w-\tilde{U})\|_{\mathrm{L}_{2}\left(I_{n} ; \mathscr{X}^{\prime}\right)} \leq C_{\tau_{n}, r_{n}} \eta_{n, 1}^{\mathrm{time}}
$$

with

$$
\eta_{n, 1}^{\mathrm{time}}:=\left\|\llbracket \Pi f-\widehat{U}^{\prime} \rrbracket_{n-1}\right\| \mathscr{X}^{\prime} .
$$

Assume further that, for every $v \in \mathscr{X}$, there exists $a \vee \in \mathbb{X}_{n}^{\ominus}$, such that

$$
\left\|\left(h_{n}^{\ominus}\right)^{-s}(v-\mathrm{V})\right\|_{\mathscr{H}} \leq C_{\text {ap }}\|v\|_{\mathscr{X}}, \quad \text { and } \quad\|\mathrm{V}\|_{\mathscr{X}} \leq C_{\text {stab }}\|v\|_{\mathscr{X}}
$$

for some $s>0$, and for generic constants $C_{\mathrm{ap}}, C_{\mathrm{stab}}>0$, independent of $v$ and of $h_{n}^{\ominus}$. Then, we also have the bound

$$
\|\mathscr{A}(w-\tilde{U})\|_{\mathrm{L}_{2}\left(I_{n} ; \mathscr{X}^{\prime}\right)} \leq C_{\tau_{n}, r_{n}} \eta_{n, 2}^{\mathrm{time}}
$$

with

$$
\eta_{n, 2}^{\text {time }}:=C_{\text {ap }}\left\|\left(h_{n}^{\ominus}\right)^{s} \llbracket \Pi f-\widehat{U}^{\prime} \rrbracket_{n-1}\right\|_{\mathscr{H}}+C_{\text {stab }}\left\|\llbracket \mathrm{U} \rrbracket_{n-1}\right\|_{\mathscr{X}}
$$


Therefore, setting $\eta_{n}^{\mathrm{time}}:=\min \left\{\eta_{n, 1}^{\mathrm{time}}, \eta_{n, 2}^{\mathrm{time}}\right\}$, we have the combined estimate

$$
\|\mathscr{A}(w-\tilde{U})\|_{\mathrm{L}_{2}\left(I_{n} ; \mathscr{X}^{\prime}\right)} \leq C_{\tau_{n}, r_{n}} \eta_{n}^{\mathrm{time}}
$$

Proof Recalling (3.6) and using the fact that the elliptic operator $\mathscr{A}$ is time independent, we immediately observe that

$$
\mathscr{A} w=\widehat{\mathscr{A} \widetilde{U}}
$$

Therefore, by Proposition 3.4 and of Remark 3.9, for $n=1, \ldots, N-1$, we conclude that

$$
C_{\tau_{n}, r_{n}}^{-1}\|\mathscr{A}(w-\widetilde{U})\|_{\mathrm{L}_{2}\left(I_{n} ; \mathscr{X}^{\prime}\right)}=\|\llbracket \mathscr{A} \widetilde{U} \rrbracket n-1\|_{\mathscr{X}^{\prime}}=\left\|\llbracket \Pi f-\widehat{U}^{\prime} \rrbracket_{n-1}\right\|_{\mathscr{X}^{\prime}},
$$

which is (5.4). Furthermore, exploit the orthogonality identity

$$
\left\langle\llbracket \mathscr{A} \tilde{U} \rrbracket_{n-1} \mid \mathrm{v}\right\rangle=\left(\llbracket A_{n} \mathrm{U} \rrbracket_{n-1}, \mathrm{v}\right)_{\mathscr{H}}=\left\langle\llbracket \mathscr{A} \mathrm{U} \rrbracket_{n-1} \mid \mathrm{v}\right\rangle \text { for all } \mathrm{v} \in \mathbb{X}_{n}^{\ominus}
$$

which follows from the definition of $\widetilde{U}$ and the properties of $\Pi$. From the latter, together with Remark 3.9, and the temporal independence of $\mathscr{A}$, we have

$$
\left\langle\llbracket \mathscr{A} \tilde{U} \rrbracket_{n-1} \mid v\right\rangle=\left(\llbracket \Pi f-\widehat{\mathrm{U}}^{\prime} \rrbracket_{n-1}, v-\mathrm{v}\right)_{\mathscr{H}}+\left\langle\mathscr{A} \llbracket \mathrm{U} \rrbracket_{n-1} \mid \mathrm{v}\right\rangle \text { for all } \mathrm{v} \in \mathbb{X}_{n}^{\ominus}
$$

From (5.6) and the continuity of $\mathscr{A}$, therefore, we deduce

$$
\left\langle\llbracket \mathscr{A} \tilde{U} \rrbracket_{n-1} \mid v\right\rangle \leq\left(C_{\text {ap }}\left\|\left(h_{n}^{\ominus}\right)^{s} \llbracket \Pi f-\widehat{U}^{\prime} \rrbracket_{n-1}\right\|_{\mathscr{H}}+C_{\text {stab }}\left\|\llbracket \mathrm{U} \rrbracket_{n-1}\right\| \mathscr{X}\right)\|v\|_{\mathscr{X}},
$$

and the result already follows.

5.3 Remark (Practical upper bound for $\eta_{n, 1}^{\text {time }}$ ) In standard settings, e.g., in the canonical example of the heat equation, cf. Example 2.2, for which (5.6) holds, we can use $\eta_{n, 2}^{\text {time }}$ for $\eta_{n}^{\text {time }}$; this is particularly pertinent when the dimension of $\mathbb{X}_{n}^{\ominus}$ is comparable to those of $\mathbb{X}_{n}$ and $\mathbb{X}_{n-1}$. In cases, however, in which (5.6) may not be effective, or even known, we may be forced to revert to (5.4); see [8] for two such settings, where one involves virtual element methods, and the other incorporates moving mesh methods. The dual norm in $\eta_{n, 1}^{\text {time }}$ typically requires a global inversion to be evaluated, in the absence of a natural Galerkin orthogonality property like (5.12) above.

Although solving an elliptic problem to determine/estimate the time estimator $\eta_{n, 1}^{\text {time }}$ may appear to be computationally demanding, such global solves may be typically performed in a lower dimensional space than $\mathbb{X}_{n}$ itself, through an a posteriori error controlled fashion, as we now demonstrate. Let $\varphi \in \mathscr{X}$ be the solution to the problem $\mathscr{A} \varphi=w$ (with $w:=\llbracket \Pi f-\widehat{U}^{\prime} \rrbracket_{n-1}$ for instance). Let also an approximation $\Phi \in \tilde{\mathbb{X}}_{n}$ to $\varphi$, for some Galerkin space $\tilde{\mathbb{X}}_{n}$, be given by

$$
\langle\mathscr{A} \Phi \mid \mathrm{v}\rangle=\langle w \mid \mathrm{v}\rangle \quad \text { for all } \mathrm{v} \in \tilde{\mathbb{X}}_{n}
$$


Now, the continuity of $\mathscr{A}$ implies $\|w\|_{\mathscr{X}^{\prime}} \leq \beta\|\varphi\|_{\mathscr{X}}$. In addition, from coercivity, continuity and the Galerkin orthogonality $\langle\mathscr{A}(\varphi-\Phi) \mid \Phi\rangle=0$, we have, respectively,

$\alpha\|\varphi\|_{\mathscr{X}}^{2} \leq\langle\mathscr{A} \varphi \mid \varphi\rangle=\langle\mathscr{A}(\varphi-\Phi) \mid \varphi-\Phi\rangle+\langle\mathscr{A} \Phi \mid \Phi\rangle \leq \beta\left(\|\varphi-\Phi\|_{\mathscr{X}}^{2}+\|\Phi\|_{\mathscr{X}}^{2}\right)$,

using also the self-adjointness of $\mathscr{A}$. The above, together with the assumed availability of an a posteriori error indicator in the energy norm give the computable bound

$$
\|w\|_{\mathscr{X}^{\prime}}^{2} \leq \frac{\beta^{3}}{\alpha}\left(\mathscr{E}_{\mathscr{X}, \tilde{\mathbb{X}}_{n}}^{2}[\Phi, w]+\|\Phi\|_{\mathscr{X}}^{2}\right)
$$

or, for the specific case $w=\llbracket \Pi f-\widehat{U}^{\prime} \rrbracket n-1$,

$$
\eta_{n, 1}^{\mathrm{time}} \leq \sqrt{\beta^{3} / \alpha}\left(\mathscr{E}_{\mathscr{X}, \tilde{\mathbb{X}}_{n}}^{2}\left[\Phi, \llbracket \Pi f-\widehat{U}^{\prime} \rrbracket_{n-1}\right]+\|\Phi\|_{\mathscr{X}}^{2}\right)^{1 / 2}
$$

5.4 Lemma (Space reconstruction error bound) For $t \in I_{n}, n=1, \ldots, N$, we have the bound

$$
\left\|(\widetilde{\widetilde{U}-U})^{\prime}(t)\right\| \mathscr{X}^{\prime} \leq \eta_{n}^{\text {space }}
$$

where

$$
\begin{aligned}
\eta_{n}^{\text {space }}(t):= & \mathscr{E}_{\mathscr{X}^{\prime}, \mathbb{X}_{n}}\left[\mathrm{U}^{\prime}(t),\left(A_{n} \mathrm{U}^{\prime}+\pi_{n} \widehat{\mathrm{U}}^{\prime \prime}-\widehat{\mathrm{U}}^{\prime \prime}\right)(t)\right] \\
& +\left|\varkappa_{n}(t)\right| \mathscr{E}_{\mathscr{X}^{\prime}, \mathbb{X}_{n}}\left[\mathrm{U}\left(t_{n-1}^{+}\right),\left(A_{n} \mathrm{U}+\pi_{n} \widehat{\mathrm{U}}^{\prime}-\widehat{\mathrm{U}}^{\prime}\right)\left(t_{n-1}^{+}\right)\right] \\
& +\left|\varkappa_{n}(t)\right| \mathscr{E}_{\mathscr{X}^{\prime}, \mathbb{X}_{n-1}}\left[\mathrm{U}\left(t_{n-1}^{-}\right),\left(A_{n-1} \mathrm{U}+\pi_{n-1} \widehat{\mathrm{U}}^{\prime}-\widehat{\mathrm{U}}^{\prime}\right)\left(t_{n-1}^{-}\right)\right],
\end{aligned}
$$

with $\varkappa_{n}: I_{n} \rightarrow \mathbb{R}$ from (4.16) in Remark 4.6, and the estimator is explicitly computable, via Assumption 3.7.

Proof Recalling (3.6), on $I_{n}$ we have

$$
(\widetilde{\widetilde{U}-U})^{\prime}=\widetilde{U}^{\prime}+\chi_{n}\left(\llbracket \widetilde{U} \rrbracket_{n-1}\right)-\mathrm{U}^{\prime}-\chi_{n}\left(\llbracket \mathrm{U} \rrbracket_{n-1}\right)
$$

Hence, with the notation of Remark 4.6, it follows that

$$
\left\|(\widetilde{\widetilde{U}-U})^{\prime}(t)\right\|_{\mathscr{X}^{\prime}} \leq\left\|\widetilde{U}^{\prime}-\mathrm{U}^{\prime}\right\|_{\mathscr{X}^{\prime}}+\left|\varkappa_{n}(t)\right|\left\|\llbracket \widetilde{U}-\mathrm{U} \rrbracket_{n-1}\right\|_{\mathscr{X}^{\prime}}, \quad t \in I_{n}
$$

Differentiating (3.16) with respect to $t \in I_{n}$, and recalling that the elliptic operator $\mathscr{A}$ is $t$-independent, we deduce the Galerkin orthogonality relation

$$
\left\langle\mathscr{A} \tilde{U}^{\prime}-\mathscr{A} \mathrm{U}^{\prime} \mid \mathrm{v}\right\rangle=0 \text { for each } \mathrm{v} \in \mathbb{X}_{n}
$$

We conclude that $\mathrm{U}^{\prime} \in \mathbb{X}_{n}$ is the Galerkin approximation of $\widetilde{U}^{\prime}$, where

$$
\mathscr{A} \widetilde{U}^{\prime}=A_{n} \mathrm{U}^{\prime}+\pi_{n} \widehat{\mathrm{U}}^{\prime \prime}-\widehat{\mathrm{U}}^{\prime \prime} \quad \text { for } t \in I_{n},
$$


by differentiating (3.14) with respect to $t$.

Furthermore, from the definition of the elliptic reconstruction (3.14), we have, respectively,

$$
\begin{aligned}
& \mathscr{A} \tilde{U}\left(t_{n-1}^{+}\right)=A_{n} \mathrm{U}\left(t_{n-1}^{+}\right)+\pi_{n} \widehat{U}^{\prime}\left(t_{n-1}^{+}\right)-\widehat{U}^{\prime}\left(t_{n-1}^{+}\right), \\
& \mathscr{A} \tilde{U}\left(t_{n-1}^{-}\right)=A_{n-1} \mathrm{U}\left(t_{n-1}^{-}\right)+\pi_{n-1} \widehat{U}^{\prime}\left(t_{n-1}^{-}\right)-\widehat{U}^{\prime}\left(t_{n-1}^{-}\right),
\end{aligned}
$$

implying, therefore,

$$
\begin{array}{ll}
\left\langle\mathscr{A} \widetilde{U}\left(t_{n-1}^{+}\right)-\mathscr{A} \mathrm{U}\left(t_{n-1}^{+}\right) \mid \mathrm{v}\right\rangle=0 & \text { for each } \mathrm{v} \in \mathbb{X}_{n} \\
\left\langle\mathscr{A} \tilde{U}\left(t_{n-1}^{-}\right)-\mathscr{A} \mathrm{U}\left(t_{n-1}^{-}\right) \mid \mathrm{v}\right\rangle=0 & \text { for each } \mathrm{v} \in \mathbb{X}_{n-1}
\end{array}
$$

This means that $\mathrm{U}\left(t_{n-1}^{+}\right) \in \mathbb{X}_{n}$ and $\mathrm{U}\left(t_{n-1}^{-}\right) \in \mathbb{X}_{n-1}$ are the Galerkin approximations of the first and second problem in (5.25), respectively.

Now, (5.24) together with Assumption 3.7, yield

$$
\left\|\widetilde{U}^{\prime}-\mathrm{U}^{\prime}\right\| \mathscr{X}^{\prime} \leq \mathscr{E} \mathscr{X}^{\prime}, \mathbb{X}_{n}\left[\mathrm{U}^{\prime}, A_{n} \mathrm{U}^{\prime}+\pi_{n} \widehat{U}^{\prime \prime}-\widehat{U}^{\prime \prime}\right] \text { on } I_{n} .
$$

Similarly, noting that

$$
\llbracket \tilde{U}-U \rrbracket_{n-1}=(\tilde{U}-U)\left(t_{n-1}^{+}\right)-(\tilde{U}-U)\left(t_{n-1}^{-}\right),
$$

and combining (5.25) with Assumption 3.7, leads to

$$
\begin{aligned}
\left\|\llbracket \widetilde{U}-\mathrm{U} \rrbracket_{n-1}\right\| \mathscr{X}^{\prime} \leq & \mathscr{E}_{\mathscr{X}^{\prime}, \mathbb{X}_{n}}\left[\mathrm{U}\left(t_{n-1}^{+}\right),\left(A_{n} \mathrm{U}+\pi_{n} \widehat{U}^{\prime}-\widehat{U}^{\prime}\right)\left(t_{n-1}^{+}\right)\right] \\
& +\mathscr{E}_{\mathscr{X}^{\prime}, \mathbb{X}_{n-1}}\left[\mathrm{U}\left(t_{n-1}^{-}\right),\left(A_{n-1} \mathrm{U}+\pi_{n-1} \widehat{U}^{\prime}-\widehat{U}^{\prime}\right)\left(t_{n-1}^{-}\right)\right] .
\end{aligned}
$$

The result already follows by inserting the above estimates into (5.22).

We are now ready to present the second main result of this work.

5.5 Theorem $\left(\mathrm{L}_{\infty}(I ; \mathscr{H})\right.$-norm a posteriori bound) With the notation of $\S 2$ and under Assumption 3.7, along with the assumptions of Lemmata 5.2 and 5.4, for each $n=$ $1, \ldots, N$, we have the bound

$$
\begin{aligned}
\| u & -\mathrm{U} \|_{\mathrm{L}_{\infty}\left(0, t_{n} ; \mathscr{H}\right)} \\
\leq & \eta^{\mathrm{in}}+(2 \alpha)^{-1 / 2} \eta_{n}^{\mathrm{osc}}+\max _{j=1, \ldots, n}\left\|\llbracket \mathrm{U} \rrbracket_{j-1}\right\|_{\mathscr{H}} \\
& +\left(\frac{1}{2 \alpha} \sum_{m=1}^{n} C_{\tau_{m}, r_{m}}^{2}\left(\eta_{m}^{\text {time }}\right)^{2}\right)^{1 / 2}+\left(\frac{1}{2 \alpha} \sum_{m=1}^{n} \int_{I_{m}}\left(\eta_{m}^{\text {space }}\right)^{2} \mathrm{~d} t\right)^{1 / 2} \\
& +3 \max _{j=1, \ldots, n} \sup _{I_{j}} \mathscr{E} \mathscr{H}, \mathbb{X}_{j}\left[\mathrm{U}, A_{j} \mathrm{U}+\pi_{j} \widehat{\mathrm{U}}^{\prime}-\widehat{\mathrm{U}}^{\prime}\right],
\end{aligned}
$$

with $\eta_{n}^{\text {osc }}$ and $\eta^{\text {in }}$ as in Theorem 4.4. 
Proof We start from (5.3), which upon testing with $\rho$ and integrating with respect to $t$ between $(0, t)$, along with the coercivity of $\mathscr{A},(2.8)$, gives

$$
\frac{1}{2}\|\rho(t)\|_{\mathscr{H}}^{2}+\alpha\|\rho\|_{\mathrm{L}_{2}(0, t ; \mathscr{X})}^{2} \leq \frac{1}{2}\|\rho(0)\|_{\mathscr{H}}^{2}+\int_{0}^{t}\langle\xi \mid \rho\rangle \mathrm{d} s,
$$

and, thus,

$$
\|\rho(t)\|_{\mathscr{H}}^{2} \leq\|\rho(0)\|_{\mathscr{H}}^{2}+\frac{1}{2 \alpha}\|\xi\|_{\mathrm{L}_{2}\left(0, t ; \mathscr{X}^{\prime}\right)}^{2}
$$

via standard arguments.

Now fix $n \in\{1, \ldots, N\}$ and choose $t^{\star} \in\left[0, t_{n}\right]$ such that

$$
\left\|\rho\left(t^{\star}\right)\right\|_{\mathscr{H}}=\|\rho\|_{\mathrm{L}_{\infty}\left(0, t_{n} ; \mathscr{H}\right)} .
$$

Then, letting $t=t^{\star}$ in (5.32), and taking the square root, yields

$$
\|\rho\|_{\mathrm{L}_{\infty}\left(0, t_{n} ; \mathscr{H}\right)} \leq\|\rho(0)\|_{\mathscr{H}}+(2 \alpha)^{-1 / 2}\|\xi\|_{\mathrm{L}_{2}\left(0, t_{n} ; \mathscr{X}^{\prime}\right)}
$$

It remains to bound the last term on the right-hand side of (5.33). To that end, applying (3.9), and involving Lemmata 5.2 and 5.4, results in

$$
\begin{aligned}
\|\xi\|_{\mathrm{L}_{2}\left(0, t_{n} ; \mathscr{X}^{\prime}\right)} & \leq \eta_{n}^{\mathrm{osc}}+\|\mathscr{A}(w-\widetilde{U})\|_{\mathrm{L}_{2}\left(0, t_{n} ; \mathscr{X}^{\prime}\right)}+\left\|(\widetilde{\widetilde{U}-\mathrm{U}})^{\prime}\right\|_{\mathrm{L}_{2}\left(0, t_{n} ; \mathscr{X}^{\prime}\right)} \\
& \leq \eta_{n}^{\text {osc }}+\left(\sum_{m=1}^{n} C_{\tau_{m}, r_{m}}^{2}\left(\eta_{m}^{\text {time }}\right)^{2}\right)^{1 / 2}+\left(\sum_{m=1}^{n} \int_{I_{m}}\left(\eta_{m}^{\text {space }}\right)^{2} \mathrm{~d} t\right)^{1 / 2} .
\end{aligned}
$$

The triangle inequality now gives

$$
\begin{aligned}
& \|u-\mathrm{U}\|_{\mathrm{L}_{\infty}\left(0, t_{n} ; \mathscr{H}\right)} \\
& \quad \leq\|\rho\|_{\mathrm{L}_{\infty}\left(0, t_{n} ; \mathscr{H}\right)}+\|w-\widetilde{U}\|_{\mathrm{L}_{\infty}\left(0, t_{n} ; \mathscr{H}\right)}+\|\widetilde{U}-\mathrm{U}\|_{\mathrm{L}_{\infty}\left(0, t_{n} ; \mathscr{H}\right)} .
\end{aligned}
$$

To estimate the second and third term on the right-hand side of (5.35), we apply (3.11), and the triangle inequality, to obtain

$$
\begin{aligned}
\|w-\widetilde{U}\|_{\mathrm{L}_{\infty}\left(0, t_{n} ; \mathscr{H}\right)} & =\max _{j=1, \ldots, n}\|\llbracket \widetilde{U} \rrbracket j-1\|_{\mathscr{H}} \\
& \leq \max _{j=1, \ldots, n}\left(\|\llbracket \widetilde{U}-\mathrm{U} \rrbracket j-1\|_{\mathscr{H}}+\left\|\llbracket \mathrm{U} \rrbracket_{j-1}\right\|_{\mathscr{H}}\right) .
\end{aligned}
$$

Also, with the aid of (3.19),

$$
\|\widetilde{U}-\mathrm{U}\|_{\mathrm{L}_{\infty}\left(0, t_{n} ; \mathscr{H}\right)} \leq \max _{j=1, \ldots, n} \sup _{t \in I_{j}} \mathscr{E}_{\mathscr{H}, \mathbb{X}_{j}}\left[\mathrm{U}, A_{j} \mathrm{U}+\pi_{j} \widehat{U}^{\prime}-\widehat{U}^{\prime}\right]
$$


Combining the last two estimates, we conclude

$$
\begin{aligned}
\| w & -\widetilde{U}\left\|_{\mathrm{L}_{\infty}\left(0, t_{n} ; \mathscr{H}\right)}+\right\| \widetilde{U}-\mathrm{U} \|_{\mathrm{L}_{\infty}\left(0, t_{n} ; \mathscr{H}\right)} \\
& \leq \max _{j=1, \ldots, n}\left(3 \sup _{t \in I_{j}} \mathscr{E} \mathscr{H}, \mathbb{X}_{j}\left[\mathrm{U}, A_{j} \mathrm{U}+\pi_{j} \widehat{U}^{\prime}-\widehat{U}^{\prime}\right]+\left\|\llbracket \mathrm{U} \rrbracket_{j-1}\right\| \mathscr{H}\right) .
\end{aligned}
$$

Combining the above completes the argument.

5.6 Remark (Alternative 'short-time' a posteriori error estimator) Starting from the alternative estimate

$$
\int_{0}^{t}\langle\xi \mid \rho\rangle \mathrm{d} s \leq\|\xi\|_{\mathrm{L}_{1}\left(0, t_{n} ; \mathscr{H}\right)}\|\rho\|_{\mathrm{L}_{\infty}\left(0, t_{n} ; \mathscr{H}\right)}
$$

the bound (5.31) with $t=t^{\star}$ as in the proof of Theorem 5.5 gives

$$
\|\rho\|_{\mathrm{L}_{\infty}\left(0, t_{n} ; \mathscr{H}\right)}^{2} \leq 2\|\rho(0)\|_{\mathscr{H}}^{2}+4\|\xi\|_{\mathrm{L}_{1}\left(0, t_{n} ; \mathscr{H}\right)}^{2}
$$

Triangle inequality trivially yields

$$
\begin{aligned}
\|\xi\|_{\mathrm{L}_{1}\left(0, t_{n} ; \mathscr{H}\right) \leq} & \|\Pi f-f\|_{\mathrm{L}_{1}\left(0, t_{n} ; \mathscr{H}\right)}+\|\mathscr{A}(w-\widetilde{U})\|_{\mathrm{L}_{1}\left(0, t_{n} ; \mathscr{H}\right)} \\
& +\left\|(\widehat{\widetilde{U}-U})^{\prime}\right\|_{\mathrm{L}_{1}\left(0, t_{n} ; \mathscr{H}\right) .}
\end{aligned}
$$

Now, using Hölder's inequality, recalling (5.10), and applying Proposition 3.4 and Remark 3.9, we have, respectively,

$$
\begin{aligned}
\|\mathscr{A}(w-\tilde{U})\|_{\mathrm{L}_{1}\left(0, t_{n} ; \mathscr{H}\right)}^{2} & \leq t_{n}\|\mathscr{A}(w-\tilde{U})\|_{\mathrm{L}_{2}\left(0, t_{n} ; \mathscr{H}\right)}^{2} \\
& =t_{n} \sum_{m=1}^{n} C_{\tau_{m}, r_{m}}^{2}\|\llbracket \mathscr{A} \widetilde{U} \rrbracket m-1\|_{\mathscr{H}}^{2} \\
& =t_{n} \sum_{m=1}^{n} C_{\tau_{m}, r_{m}}^{2}\left\|\llbracket \Pi f-\widehat{U}^{\prime} \rrbracket_{m-1}\right\|_{\mathscr{H}}^{2} .
\end{aligned}
$$

In addition, an inspection of the proof of Lemma 5.4 reveals the bound

$$
\left\|(\widetilde{\widetilde{U}-U})^{\prime}(t)\right\|_{\mathscr{H}} \leq \eta_{m}^{\text {space }}(t)
$$

for $t \in I_{m}$, where

$$
\begin{aligned}
\eta_{m}^{\text {space }}(t):= & \mathscr{E} \mathscr{H}, \mathbb{X}_{m}\left[\mathrm{U}^{\prime}(t),\left(A_{m} \mathrm{U}^{\prime}+\pi_{m} \widehat{U}^{\prime \prime}-\widehat{U}^{\prime \prime}\right)(t)\right] \\
& +\left|\varkappa_{m}(t)\right| \mathscr{E} \mathscr{H}, \mathbb{X}_{m}\left[\mathrm{U}\left(t_{m-1}^{+}\right),\left(A_{m} \mathrm{U}+\pi_{m} \widehat{U}^{\prime}-\widehat{U}^{\prime}\right)\left(t_{m-1}^{+}\right)\right] \\
& +\left|\varkappa_{m}(t)\right| \mathscr{E} \mathscr{H}, \mathbb{X}_{m-1}\left[\mathrm{U}\left(t_{m-1}^{-}\right),\left(A_{m-1} \mathrm{U}+\pi_{m-1} \widehat{U}^{\prime}-\widehat{U}^{\prime}\right)\left(t_{m-1}^{-}\right)\right] .
\end{aligned}
$$


Combining the above estimates, we arrive at

$$
\begin{aligned}
\|\rho\|_{\mathrm{L}_{\infty}\left(0, t_{n} ; \mathscr{H}\right) \leq} & \sqrt{2}\left\|u_{0}-\pi_{0} u_{0}\right\| \mathscr{H}+2\|\Pi f-f\|_{\mathrm{L}_{1}\left(0, t_{n} ; \mathscr{H}\right)} \\
& +2\left(t_{n} \sum_{m=1}^{n} C_{\tau_{m}, r_{m}}^{2}\left\|\llbracket \Pi f-\widehat{\mathrm{U}}^{\prime} \rrbracket m-1\right\|_{\mathscr{H}}^{2}\right)^{1 / 2} \\
& +2 \sum_{m=1}^{n} \int_{I_{m}} \eta_{m}^{\text {space }} \mathrm{d} t
\end{aligned}
$$

this, in conjunction with (5.38) now yields an alternative a posteriori error bound which may be superior to the one given in Theorem 5.5 for small final time $t_{n}$.

\section{$6 \mathbf{H}^{1}\left(\mathscr{I} ; \mathscr{X}^{\prime}\right)$-type a posteriori error estimates}

We conclude this work by briefly arguing on how our techniques allow to derive $\mathrm{H}^{1}\left(\mathscr{I} ; \mathscr{X}^{\prime}\right)$-type a posteriori error estimates. To this end, for any $\mathscr{I}$-piecewise sufficiently smooth function $z$, we define the broken (semi-)norm

$$
\|z\|_{\mathrm{H}^{1}\left(\mathscr{I} ; \mathscr{X}^{\prime}\right)}:=\left(\sum_{n=1}^{N}\left\|z^{\prime}+\chi_{n}\left(\llbracket z \rrbracket_{n-1}\right)\right\|_{\mathrm{L}_{2}\left(I_{n} ; \mathscr{X}^{\prime}\right)}^{2}\right)^{1 / 2}
$$

with $\mathscr{I}$ signifying the time partition of $I=(0, T]$ from $(2.17)$. Then, recalling $\rho$ from (5.1), the triangle inequality yields

$$
\|u-U\|_{\mathrm{H}^{1}\left(\mathscr{I} ; \mathscr{X}^{\prime}\right)} \leq|\rho|_{\mathrm{H}^{1}\left(I ; \mathscr{X}^{\prime}\right)}+\|w-\mathrm{U}\|_{\mathrm{H}^{1}\left(\mathscr{I} ; \mathscr{X}^{\prime}\right)},
$$

upon noting the continuity of $\rho$ with respect to the time variable. To control the first term on the right-hand side of (6.2), we start from (5.3), and notice that

$$
|\rho|_{\mathrm{H}^{1}\left(I ; \mathscr{X}^{\prime}\right)}=\|\xi-\mathscr{A} \rho\|_{\mathrm{L}_{2}\left(I ; \mathscr{X}^{\prime}\right)} \leq\|\xi\|_{\mathrm{L}_{2}\left(I ; \mathscr{X}^{\prime}\right)}+\beta\|\rho\|_{\mathrm{L}_{2}(I ; \mathscr{X})},
$$

with $\beta$ from (2.8). Furthermore, based on (5.31) with $t=T$, standard arguments give

$$
\alpha\|\rho\|_{\mathrm{L}_{2}(I ; \mathscr{X})}^{2} \leq\|\rho(0)\|_{\mathscr{H}}^{2}+\alpha^{-1}\|\xi\|_{\mathrm{L}_{2}\left(I ; \mathscr{X}^{\prime}\right)}^{2} .
$$

Combining the last two bounds, we arrive at

$$
|\rho|_{\mathrm{H}^{1}\left(\mathscr{I} ; \mathscr{X}^{\prime}\right)}^{2} \leq \frac{2 \beta^{2}}{\alpha}\|\rho(0)\|_{\mathscr{H}}^{2}+2\left(1+\frac{\beta^{2}}{\alpha^{2}}\right)\|\xi\|_{\mathrm{L}_{2}\left(I ; \mathscr{X}^{\prime}\right)}^{2}
$$

The first term on the right-hand side of (6.5) is bounded trivially by $\eta^{\text {in }}$ (defined in Theorem 4.4), while for the second we use (5.34). To estimate the second term on the 
right-hand side of (6.2), we consider the splitting

$$
\|w-U\|_{\mathrm{H}^{1}\left(\mathscr{I} ; \mathscr{X}^{\prime}\right)} \leq\|w-\widehat{\mathrm{U}}\|_{\mathrm{H}^{1}\left(\mathscr{I} ; \mathscr{X}^{\prime}\right)}+\|\widehat{\mathrm{U}}-\mathrm{U}\|_{\mathrm{H}^{1}\left(\mathscr{I} ; \mathscr{X}^{\prime}\right)} .
$$

The second term on the right-hand side of the last estimate is a computable quantity, while the first can be immediately bounded using Lemma 5.4, through the linearity of the time reconstruction.

\section{Conclusions}

In this article we have presented a posteriori error estimates for fully discrete $h p$ DG-in-time and general Galerkin-in-space discretizations of abstract linear parabolic PDE. Our approach is based on a novel combination of temporal and elliptic reconstructions, the latter allowing for arbitrary elliptic error spatial estimators. Our main results include computable $\mathrm{L}_{\infty}(\mathscr{H})$ - and $\mathrm{L}_{2}(\mathscr{X})$-a posteriori error estimates; some remarks concerning $\mathrm{H}^{1}\left(\mathscr{X}^{\prime}\right)$-norm error estimation are given as well. Finally, we note that a series of numerical experiments showcasing the optimality of the a posteriori error estimators derived above are given in [7].

Acknowledgements We would like to thank the referees for their thoughtful remarks that have led to relevant improvements of our results. EHG acknowledges financial support by The Leverhulme Foundation (Grant No. RPG-2015-306) and by the Marie Skłodowska-Curie ITN "ModCompShock". OL's contribution was partially supported by the Marie Skłodowska-Curie ITN "ModCompShock". TPW acknowledges the financial support of the Swiss National Science Foundation (SNF), Grant No. 200021_182524.

Open Access This article is licensed under a Creative Commons Attribution 4.0 International License, which permits use, sharing, adaptation, distribution and reproduction in any medium or format, as long as you give appropriate credit to the original author(s) and the source, provide a link to the Creative Commons licence, and indicate if changes were made. The images or other third party material in this article are included in the article's Creative Commons licence, unless indicated otherwise in a credit line to the material. If material is not included in the article's Creative Commons licence and your intended use is not permitted by statutory regulation or exceeds the permitted use, you will need to obtain permission directly from the copyright holder. To view a copy of this licence, visit http://creativecommons.org/licenses/by/4.0/.

\section{References}

1. Akrivis, G., Makridakis, C., Nochetto, R.H.: A posteriori error estimates for the Crank-Nicolson method for parabolic equations. Math. Comp. 75(254), 511-531 (2006)

2. Ainsworth, M., Tinsley Oden, J.: A posteriori error estimation in finite element analysis. Pure and Applied Mathematics (New York). Wiley, New York (2000)

3. Braess, D., Pillwein, V., Schöberl, J.: Equilibrated residual error estimates are p-robust. Comput. Methods Appl. Mech. Eng. 198(13-14), 1189-1197 (2009)

4. Braess, D.: Finite elements: theory, fast solvers, and applications in elasticity theory, 3rd edn, p. 4. Cambridge University Press, Cambridge (2007)

5. Brenner, S.C., Scott, L.R.: The mathematical theory of finite element methods. Texts in Applied Mathematics, 3rd edn. Springer, New York (2007)

6. Cangiani, A., Georgoulis, E.H., Metcalfe, S.: Adaptive discontinuous Galerkin methods for nonstationary convection-diffusion problems. IMA J. Numer. Anal. 34(4), 1578-1597 (2014) 
7. Cangiani, A., Georgoulis, E.H., Sabawi, M.: A posteriori error analysis for implicit-explicit $h p$ discontinuous Galerkin timestepping methods for semilinear parabolic problems. J. Sci. Comput. 82(2), 24 (2020)

8. Cangiani, Andrea, Georgoulis, Emmanuil H., Sutton, Oliver J.: Adaptive non-hierarchical Galerkin methods for parabolic problems with application to moving mesh and virtual element methods. Online preprint arXiv:2005.05661, May (2020)

9. Destuynder, P., Métivet, B.: Explicit error bounds in a conforming finite element method. Math. Comput. 68(228), 1379-1396 (1999)

10. Eriksson, K., Johnson, C.: Adaptive finite element methods for parabolic problems. I. A linear model problem. SIAM J. Numer. Anal. 28(1), 43-77 (1991)

11. Eriksson, K., Johnson, C.: Adaptive finite element methods for parabolic problems. II. Optimal error estimates in $L_{\infty} L_{2}$ and $L_{\infty} L_{\infty}$. SIAM J. Numer. Anal. 32(3), 706-740 (1995)

12. Eriksson, K., Johnson, C.: Adaptive finite element methods for parabolic problems. IV. Nonlinear problems. SIAM J. Numer. Anal. 32(6), 1729-1749 (1995)

13. Eriksson, K., Johnson, C.: Adaptive finite element methods for parabolic problems. V. Long-time integration. SIAM J. Numer. Anal. 32(6), 1750-1763 (1995)

14. Eriksson, K., Johnson, C., Larsson, S.: Adaptive finite element methods for parabolic problems. VI. Analytic semigroups. SIAM J. Numer. Anal. 35(4), 1315-1325 (1998). (electronic)

15. Eriksson, K., Johnson, C., Thomée, V.: Time discretization of parabolic problems by the discontinuous Galerkin method. RAIRO Modél. Math. Anal. Numér. 19(4), 611-643 (1985)

16. Ern, A., Smears, I., Vohralík, M.: Guaranteed, locally space-time efficient, and polynomial-degree robust a posteriori error estimates for high-order discretizations of parabolic problems. SIAM J. Numer. Anal. 55(6), 2811-2834 (2017)

17. Ern, A., Smears, I., Vohralík, M.: Equilibrated flux a posteriori error estimates in $L^{2}\left(H^{1}\right)$-norms for high-order discretizations of parabolic problems. IMA J. Numer. Anal. 39(3), 1158-1179 (2019)

18. Georgoulis, E.H., Makridakis, C.: On a posteriori error control for the Allen-Cahn problem. Math. Methods Appl. Sci. 37(2), 173-179 (2014)

19. Gaspoz, F.D., Siebert, K., Kreuzer, C., Ziegler, D.A.: A convergent time-space adaptive dG $(s)$ finite element method for parabolic problems motivated by equal error distribution. IMA J. Numer. Anal. 39(2), 650-686 (2019)

20. Holm, B., Wihler, T.P.: Continuous and discontinuous Galerkin time stepping methods for nonlinear initial value problems with application to finite time blow-up. Numer. Math. 138(3), 767-799 (2018)

21. Jamet, P.: Galerkin-type approximations which are discontinuous in time for parabolic equations in a variable domain. SIAM J. Numer. Anal. 15(5), 912-928 (1978)

22. Kyza, I., Metcalfe, S., Wihler, T.P.: $h p$-adaptive Galerkin time stepping methods for nonlinear initial value problems. J. Sci. Comput. 75(1), 111-127 (2018)

23. Lakkis, O., Makridakis, C.: Elliptic reconstruction and a posteriori error estimates for fully discrete linear parabolic problems. Math. Comp. 75(256), 1627-1658 (2006)

24. Lakkis, Omar: Makridakis, Charalambos: Elliptic reconstruction and a posteriori error estimates for fully discrete linear parabolic problems. Math. Comp. 75(256), 1627-1658 (2006)

25. Makridakis, C., Babuška, I.: On the stability of the discontinuous Galerkin method for the heat equation. SIAM J. Numer. Anal. 34(1), 389-401 (1997)

26. Makridakis, C., Nochetto, R.H.: Elliptic reconstruction and a posteriori error estimates for parabolic problems. SIAM J. Numer. Anal. 41(4), 1585-1594 (2003)

27. Makridakis, C., Nochetto, R.H.: A posteriori error analysis for higher order dissipative methods for evolution problems. Numer. Math. 104(4), 489-514 (2006)

28. Makridakis, Charalambos, Nochetto, Ricardo H.: A posteriori error analysis for higher order dissipative methods for evolution problems. Numer. Math. 104(4), 489-514 (2006)

29. Matache, A.-M., Schwab, C., Wihler, T.P.: Fast numerical solution of parabolic integrodifferential equations with applications in finance. SIAM J. Sci. Comput. 27, 369-393 (2005)

30. Matache, A.-M., Schwab, C., Wihler, T.P.: Linear complexity solution of parabolic integro-differential equations. Numer. Math. 104, 69-102 (2006)

31. Picasso, M.: Adaptive finite elements for a linear parabolic problem. Comput. Methods Appl. Mech. Engrg. 167(3-4), 223-237 (1998)

32. Roubíček, T.: Nonlinear partial differential equations with applications. International Series of Numerical Mathematics, 2nd edn. Birkhäuser/Springer Basel AG, Basel (2013) 
33. Schötzau, D., Schwab, C.: Time discretization of parabolic problems by the $h p$-version of the discontinuous Galerkin finite element method. SIAM J. Numer. Anal. 38, 837-875 (2000)

34. Schötzau, D., Schwab, C.: $h p$-discontinuous Galerkin time-stepping for parabolic problems. C. R. Acad. Sci. Paris, Série I 333, 1121-1126 (2001)

35. Schötzau, D., Wihler, T.P.: A posteriori error estimation for $h p$-version time-stepping methods for parabolic partial differential equations. Numer. Math. 115(3), 475-509 (2010)

36. Verfürth, R.: A posteriori error estimates for nonlinear problems $L^{r}\left(0, T ; L^{\rho}(\Omega)\right)$-error estimates for finite element discretizations of parabolic equations. Math. Comp. 67(224), 1335-1360 (1998)

37. Verfürth, R.: A posteriori error estimates for finite element discretizations of the heat equation. Calcolo 40(3), 195-212 (2003)

38. von Petersdorff, T., Schwab, C.: Numerical solution of parabolic equations in high dimensions. Math. Model. Anal. Numer. 38, 93-127 (2004)

39. Werder, T., Gerdes, K., Schötzau, D., Schwab, C.: hp-discontinuous Galerkin time-stepping for parabolic problems. Comput. Methods Appl. Mech. Eng. 190, 6685-6708 (2001)

Publisher's Note Springer Nature remains neutral with regard to jurisdictional claims in published maps and institutional affiliations. 